\title{
Free Fall Mechanism of Micro Liquid Droplet
}

\author{
Tetsuya Ono and Akira Kawai* \\ Department of Electrical Engineering, Nagaoka University of Technology, \\ 1603-1 Kamitomioka, Nagaoka, Niigata 940-2188, Japan \\ (*Corresponding author,kawai@nagaokaut.ac.jp)
}

\begin{abstract}
It is important to analyze a behavior of micro liquid droplet in order to achieve a high accuracy of LSI fabrication process such as the cleaning process, the lithography process and the etching process, patterning process and so on. By using a high-speed camera, free fall behavior of a droplet is observed. The Laplace force before falling acts to a droplet effectively. By observing the position of droplet head and tail, it is oberved that the micro droplet falls faster than the free fall velocity. By employing a droplet spring vibration system, the fall nature can be clarified.
\end{abstract}

Keywords: Micro droplet, Laplace, free fall, ink jet, surface tension

\section{Introduction}

Recently, wetting control is one important process in micro device fabrication. Particularly, micro liquid droplet control has been focused in order to achieve high precise process control such as inkjet printing and nozzle jet coating and so on. [1-4]. In this regard, we pay attention to droplet fall behavior as a basic dispense process. By using a high-speed camera, slight vibration of droplet under hanging up by meniscus neck and fall velocity faster than free fall can be confirmed. The double spring model is employed to discuss the droplet fall mechanism.

\section{Experiment}

A schematic of the experimental setup is shown in Fig.1a. In the experiment, as a falling water droplet, deionized water $\left(\gamma=72.8 \mathrm{~mJ} / \mathrm{m}^{2}\right)$ was used. The nozzle diameter for droplet injection was $720 \mu \mathrm{m}$. A high-speed camera (VCC-8000,Digimo) was used for the observation of droplet falling. With the image resolution of $256 \times 212$ pixels in $1.4 \mathrm{~cm}^{2}$ area, the system can record droplet images at 1000 frames/sec. As shown in Fig.1b, the droplet head and tail positions from the nozzle apex were measured. The position of $y=0 \mathrm{~mm}$ can be defined as nozzle apex. All experiments were carried out at the ambient conditions, $20.0^{\circ} \mathrm{C}$ and $30 \% \mathrm{RH}$.

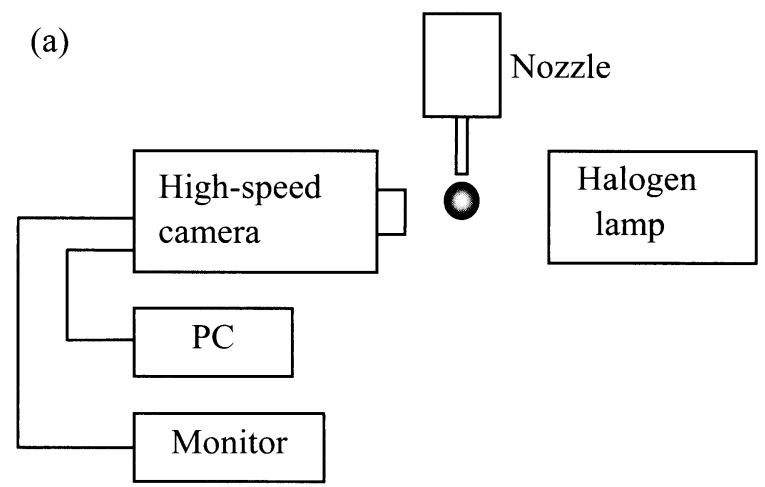

(b)

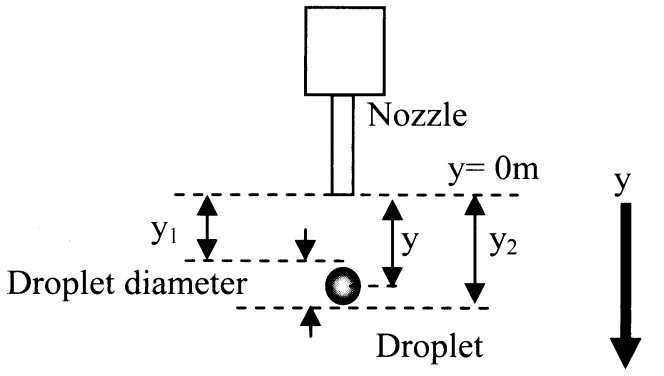

Fig.1 Experimental setup; (a) the experimental apparatus, (b) Definition of droplet position after drop from nozzle apex. 


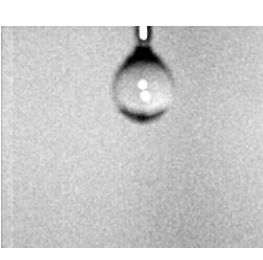

$0 \mathrm{~ms}$

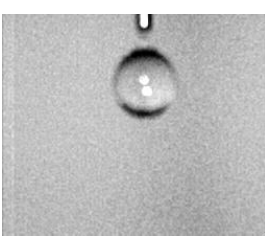

$25 \mathrm{~ms}$

$\left(\mathrm{t}_{1}=0\right)$

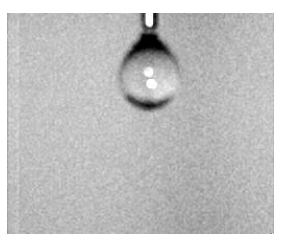

$5 \mathrm{~ms}$

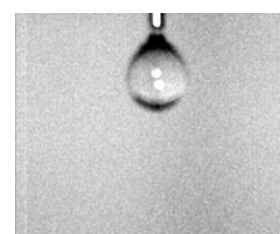

$10 \mathrm{~ms}$

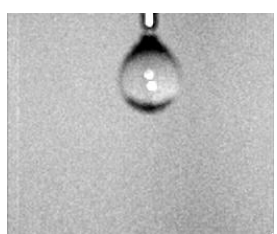

$15 \mathrm{~ms}$

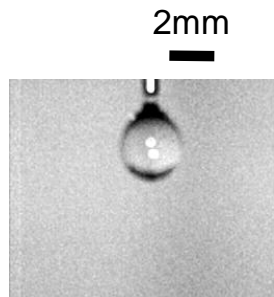

$20 \mathrm{~ms}$

(a) Before separation ( I )

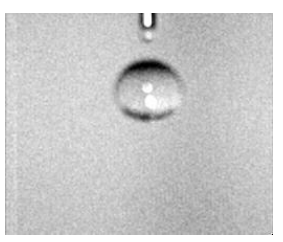

$30 \mathrm{~ms}$

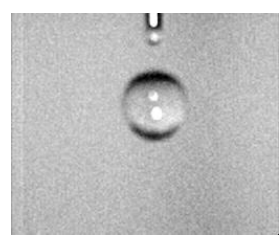

$35 \mathrm{~ms}$

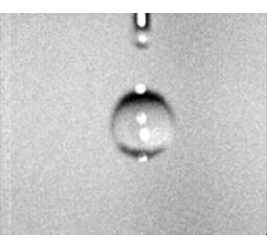

$40 \mathrm{~ms}$

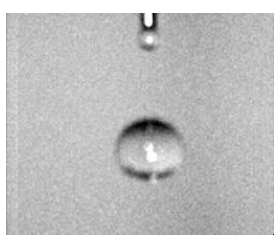

$45 \mathrm{~ms}$

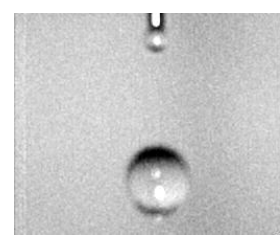

$50 \mathrm{~ms}$

(b) After separation (II)

Fig.2 Sequential images of drolet falling. The time $t_{1}$ is defined as a moment at droplet separation from nozzle apex.

\section{Results and Discussion}

We can obtain the observation results of drolet falling as shown in Fig.2. The time $t_{1}=0 \mathrm{~s}$ can be defined as the separation moment from a nozzle apex. The images of free droplet until separation ( I ) and droplet falling behavior (II) are recorded for every $5 \mathrm{~ms}$ by using the high speed camera. The droplet diameter was $3.06 \mathrm{~mm}$ at $t_{l}=0 \mathrm{~s}$. The droplet is hung by the slight water meniscus. Figure 3 shows the droplet positions, head and tail, as time passage. The symbols $\mathrm{y}_{1}$ and $\mathrm{y}_{2}$ are defined as droplet tail and head positions as shown Fig.1b. Before falling ( I ), the droplet position increases gradually, which means the tension of water meniscus. The Laplace force acting on the water

Time t $[\mathrm{ms}]$

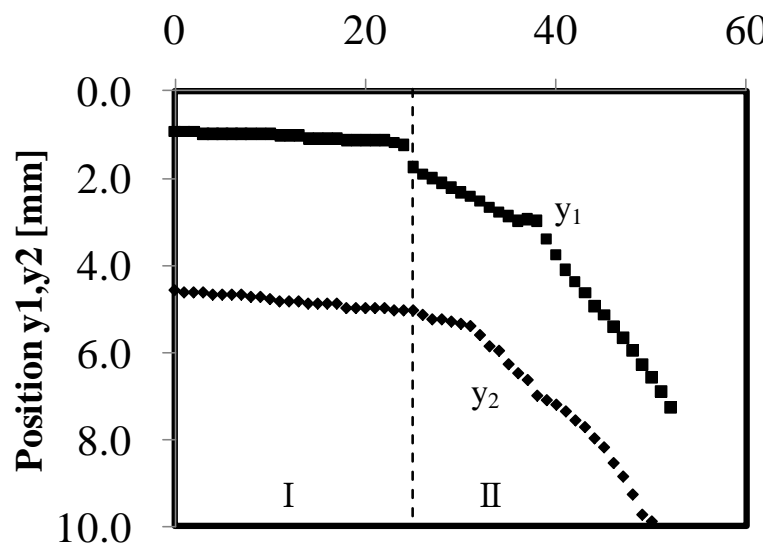

Fig.3 The Droplet positions at head and tail as the passage time. meniscus is defined as the following equation.[6]

$$
\begin{aligned}
F=\pi R_{0}{ }^{2} \gamma & \sin ^{2} \alpha\left(\frac{1}{R_{1}}-\frac{1}{R_{2}}\right) \\
& +2 \pi R_{0} \gamma \sin \alpha \sin (\alpha+\theta)
\end{aligned}
$$

where symbols $F, \gamma$ and $R_{0}$ represent Laplace force, surface energy and radius of droplet as shown in Fig.4. The symbols $\theta$ and $\alpha$ represent the conact angle of the menicus and the center angle of droplet. The radius $R_{I}$ and $R_{2}$ indicate the curvature radius of meniscus. In Fig.4a, the following values are obtained, $R_{0}=1.5 \mathrm{~mm}, R_{1}=1.0 \mathrm{~mm}, R_{2}=0.85$ $\mathrm{mm}, \alpha=5^{\circ}, \theta=27.8^{\circ}$. The Lalace force $\mathrm{F}$ acting on water droplet is estimated to be $0.086 \mathrm{mN}$.

We focus on the droplet fall region II in Fig.3. The droplet head and tail positions are plotted in

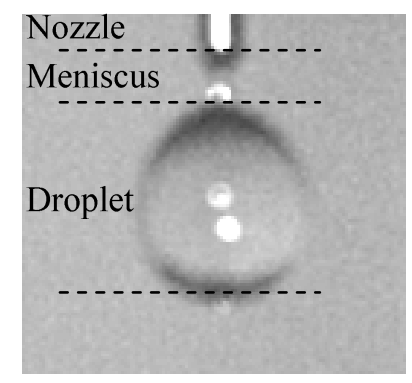

(a) Photograph

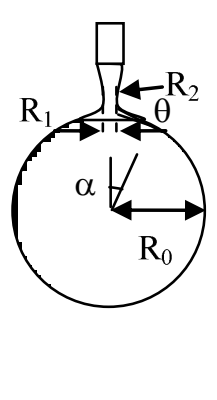

(b) Definition
Fig.4 Analysis model of Laplace force based on meniscus shape of water droplet. 
Fig.5. The data indicate the center position of the droplet. We attempted to analyze the free fall behavior of droplet without air resistance. The symbols of $y_{g}$ and $y_{g 0}$ are defined as the positions of droplet in free falling and initial position of droplet at $t_{1}=0 \mathrm{~s}$. The following motion equation can be defined.

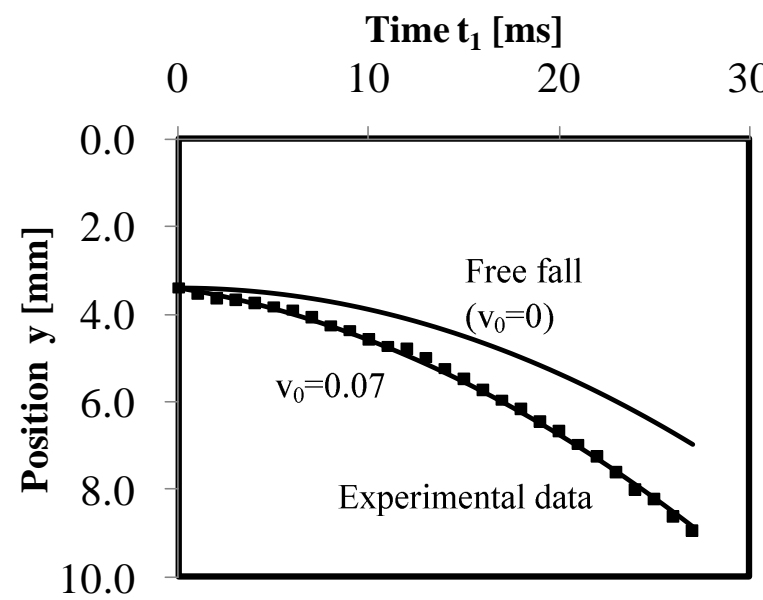

(a) Droplet position at passage time.

Time $\mathbf{t}_{\mathbf{1}}[\mathrm{ms}]$

1

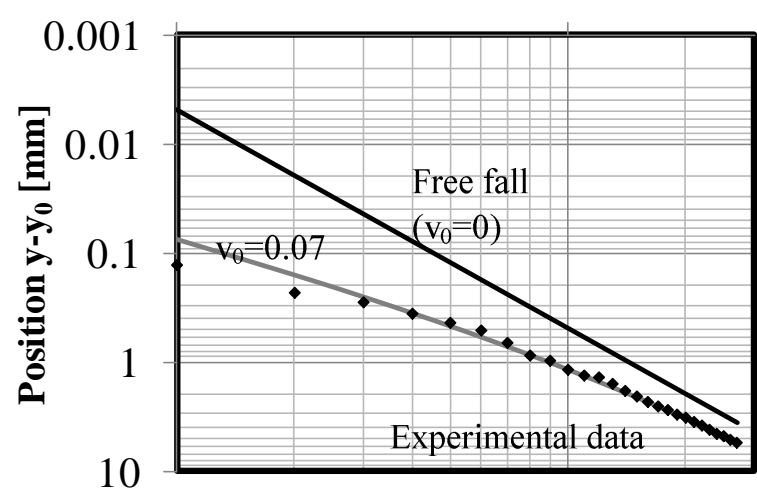

(b) Droplet position and passage time (logarithm).

Fig.5 Difference of water droplet falling from free fall motion.

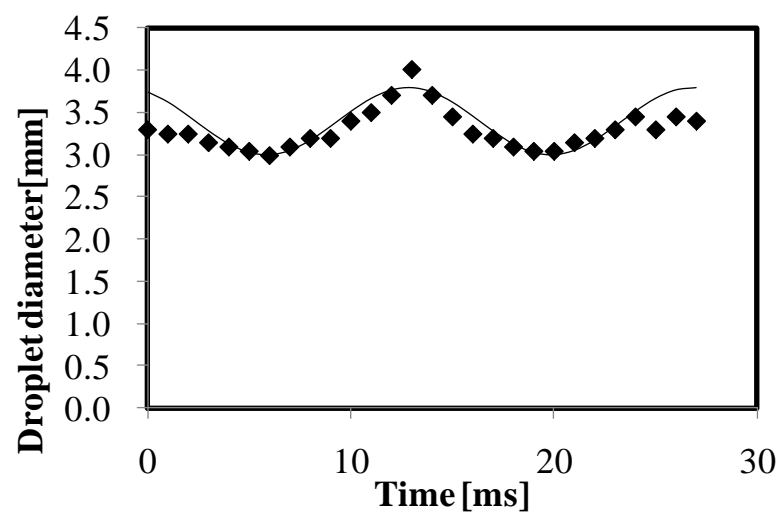

Fig.6 Vibration of droplet size during falling.

$$
y_{g}-y_{g 0}=\frac{1}{2} g t_{1}^{2}
$$

where the symbol $g$ represents acceleration due to gravity. The free fall behavior is shown in Fig.5a as a solid curve. It is cleary observed that the experimental data of water droplet falling is differ from the theoretical free fall nature. The water droplet falls faster than that under free fall. In this regard, an initial velocity $\mathrm{v}_{0}$ at $\mathrm{t}_{1}=0$ of the droplet is employed as the following equation.

$$
y_{g}-y_{g 0}=\frac{1}{2} g t_{1}^{2}+\mathrm{v}_{0} t_{1}
$$

By the fitting with experimental data, the iniital velocity can be estimated to be $0.07 \mathrm{~m} / \mathrm{s}$.

Then, we focus on the droplet nature in more detail. Figure 6 shows the variation of the falling droplet diameter observed in Fig.2. It is observed clearly that the water droplet vibrates slightly and changes its size during fall. Based on the observation data, assuming that variation of the droplet diameter is simple harmonic motion. Therefore, the following formula can be considered.

$$
x=A_{v} \sin \left(\omega t_{1}+\phi_{0}\right)+x_{0}
$$

where symbol $x$ is droplet diameter, $A_{v}$ is amplitude parameter, $\omega$ is angular frequency, $t_{1}$ is time, $\phi_{0}$ and $x_{0}$ are initial phase and position. By the fitting with experimental data, the $\omega$ value is obtained as 448.7 $\mathrm{rad} / \mathrm{s}$. Hence, the spring constant of a droplet can be estimated. The $\omega$ in Eq. 4 at simple harmonic motion is defined as follows.

$$
\omega_{2}=\sqrt{\frac{k_{2}}{m_{2}}}
$$

where symbols $\omega_{2}$ and $k_{2}$ represent the angular frequency and spring constant of the water droplet. Volume of the droplet calculated as $1.5 \mathrm{ml}$ from the photograph. Density of water was $998.2 \mathrm{kgm}^{-3}$ at

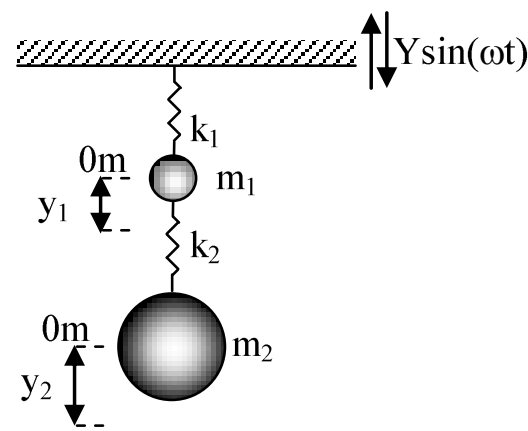

Fig.7 Schematic illustration of double spring model composed with meniscus and droplet. 
$20.0^{\circ} \mathrm{C}$. Consequentially, the spring constant $k_{2}$ of water droplet can be determined to be $2.83 \mathrm{~N} / \mathrm{m}$. In order analyze the falling nature of water droplet, it is employed that the double spring vibrating system as shown Fig.7. The water meniscus and droplet are regardedas certain springs. The equation of motion of this model can be expressd based on the force balance as follows.

$$
\left\{\begin{array}{l}
m_{1} \frac{d^{2} y_{1}}{d t^{2}}+k_{2}\left(y_{1}-y_{2}\right)+k_{1}\left(y_{1}-Y \sin (\omega t)\right)=0 \\
m_{2} \frac{d^{2} y_{2}}{d t^{2}}-k_{2}\left(y_{2}-y_{1}\right)=0
\end{array}\right.
$$
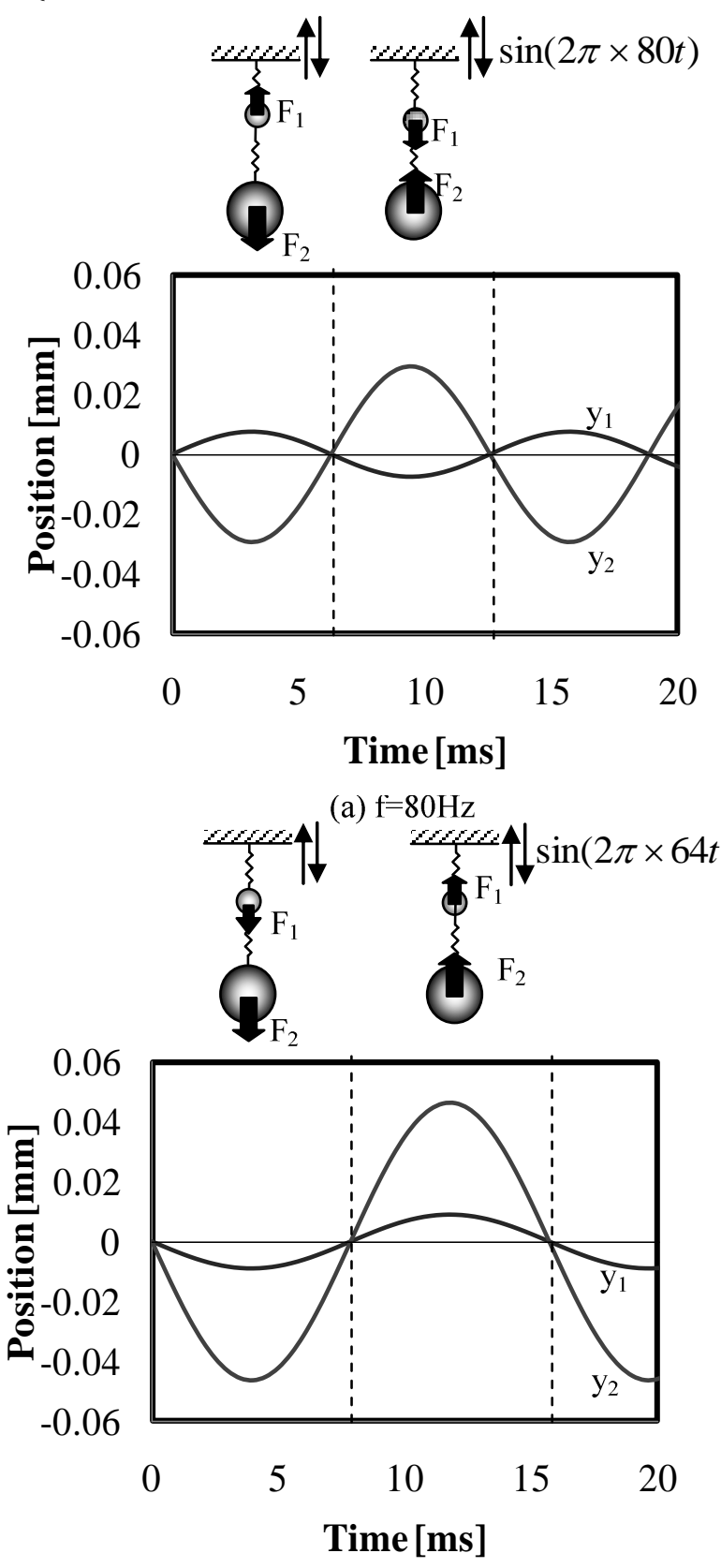

(b) $\mathrm{f}=64 \mathrm{~Hz}$

Fig.8 Dependency of position and time.
Solving the above equation.

$$
\left\{\begin{array}{l}
y_{1}=\frac{\left(-\omega^{2}+\omega_{12}{ }^{2}\right) \omega_{11}{ }^{2} Y \sin \omega t}{\omega^{4}-\omega^{2}\left(\omega_{11}{ }^{2}+\omega_{12}{ }^{2}+\omega_{21}{ }^{2}\right)+\omega_{11}{ }^{2} \omega_{12}{ }^{2}} \\
y_{2}=\frac{\omega_{11}{ }^{2} \omega_{12}{ }^{2} Y \sin \omega t}{\omega^{4}-\omega^{2}\left(\omega_{11}{ }^{2}+\omega_{12}{ }^{2}+\omega_{21}{ }^{2}\right)+\omega_{11}{ }^{2} \omega_{12}{ }^{2}}
\end{array}\right.
$$

where $\omega_{11}=\sqrt{k_{1} / m_{1}}, \omega_{12}=\sqrt{k_{1} / m_{2}}, \omega_{22}=\sqrt{k_{2} / m_{2}}$. The meniscus spring constant $\mathrm{k}_{1}$ is obtained based on Hooke's low with the volume of the meniscus bridge, $0.2 \mu \mathrm{l}$, from the observed image and the Laplace force $0.086 \mathrm{mN}$ from Eq.1. Based on Eq.7, the vibrating motion of water droplet and meniscus are shown in Fig.8. The vibration frequency of water droplet is determined as $71.4 \mathrm{~Hz}$ from Fig.6. In Fig.8, the positions of water meniscus $y_{1}$ and droplet $\mathrm{y}_{2}$ are estimated at the two representative frequency 80 and $64 \mathrm{~Hz}$. In the initial stage less than $6 \mathrm{~ms}$ in $80 \mathrm{~Hz}$ and $8 \mathrm{~ms}$ in $64 \mathrm{~Hz}$, the downward force $F_{2}$ acts to water droplet. The same mode of vibration is repeated periodically. It can be considered that the water droplet should be separated in this mode. Moreover, the downward force is added to the falling droplet. This is the reason of that the water droplet falls faster than free fall one as shown in Fig.5.

\section{Conclusion}

We critically analyzed the behavior of micro water droplet by the high speed camera measurements. The mechanism of droplet falling is explaind by employing the double spring model. The obtained results in this paper can be applied to various industrial processes controlling the micro liquid drops.

\section{References}

1. R. Shah, D. Merreceyes, M. Husemann, I. Rees, N. Abbott, C. Hawker, J. Hedrick, Macromolecules, 33 (2000) 597.

2. E. Ide, S. Angate, A. Hirose, K. Kobayashi, Acta Materilia 53 (2005) 2385.

3. D. Wakuda, S. Suganuma, Chem. Phys. Letter, 441 (2007) 305.

4. T. Lee, T. Kang, J. Yang, J. Jo, K. Kim, B. Choi, D. Kim, J. Manufacturing Science Engineering 130 (2008) 03111315.

5. W.pietsch and H.Rumpf, Chem. Ind. Technol. 18 (1967) 885.

6. J. N Israelachivili, Intermolecular and Surface Force, $2^{\text {nd }}$ Edition, (Academic Press). 